Article

\title{
Identification of MicroRNA Targeting Mlph and Affecting Melanosome Transport
}

\author{
Jeong Ah Lee ${ }^{\dagger}$, Seok Joon Hwang ${ }^{\dagger}$, Sung Chan Hong, Cheol Hwan Myung, Ji Eun Lee, \\ Jong Il Park and Jae Sung Hwang *(D) \\ Department of Genetic Engineering \& Graduate School of Biotechnology, Kyung Hee University, Yongin, \\ Gyeonggi-do 446-701, Korea \\ * Correspondence: jshwang@khu.ac.kr; Tel.: +82-312-013-797 \\ + Equal contribution.
}

Received: 27 June 2019; Accepted: 6 July 2019; Published: 8 July 2019

\begin{abstract}
Melanosomes undergo a complex maturation process and migrate into keratinocytes. Melanophilin (Mlph), a protein complex involving myosin Va (MyoVa) and Rab27a, enables the movement of melanosomes in melanocytes. In this study, we found six miRNAs targeting Mlph in mouse using two programs (http://targetscan.org and DianaTools). When melan-a melanocytes were treated with six synthesized microRNAs, miR-342-5p, miR-1839-5p, and miR-3082-5p inhibited melanosome transport and induced melanosome aggregation around the nucleus. The other microRNAs, miR-5110, miR-3090-3p, and miR-186-5p, did not inhibit melanosome transport. Further, miR-342-5p, miR-1839-5p, and miR-3082-5p decreased Mlph expression. The effect of miR-342-5p was the strongest among the six synthesized miRNAs. It inhibited melanosome transport in melan-a melanocytes and reduced Mlph expression in mRNA and protein levels in a dose-dependent manner; however, it did not affect Rab27a and MyoVa expressions, which are associated with melanosome transport. To examine miR-342-5p specificity, we performed luciferase assays in a mouse melanocyte-transfected reporter vector including Mlph at the $3^{\prime}$-UTR (untranslated region). When treated with miR-342-5p, luciferase activity that had been reduced by approximately $50 \%$ was restored after inhibitor treatment. Therefore, we identified a novel miRNA affecting Mlph and melanosome transport, and these results can be used for understanding Mlph expression and skin pigmentation regulation.
\end{abstract}

Keywords: melanosome transport; melanophilin; microRNA

\section{Introduction}

Melanocytes, a type of skin cell, produce melanin and are located in the basal layer of the epidermis. Melanin is a biological pigment comprising the reddish-yellow type called pheomelanin and the brownish-black type eumelanin [1,2]. The differences in skin color among individuals from different races result from the type of melanin produced and not from the number of melanocytes [3].

Melanosomes are lysosome-related organelles that produce, store, and transport melanin, and result in the pigmentation in melanocytes [4]. The transport of mature melanosomes from the perinuclear area to the dendrite tips of melanocytes is facilitated by microtubule- and actin-dependent motor proteins [5]. Actin-dependent melanosome transport is associated with the tripartite complex proteins, Rab27a, melanophilin (Mlph), and myosin Va (MyoVa). Rab27a is a GTPase and a member of the Ras oncogene family, and its effector molecule is Slac2-a/Mlph, which recruits the actin-dependent motor protein MyoVa [5-7]. Defects in melanosome transport cause a condition known as Griscelli syndrome (GS). Griscelli and colleagues found two girls with skin hypopigmentation and silver-gray hair [8]. There are three types of GS, GS types 1, 2, and 3, caused by mutations in MyoVa, Rab27a, 
and Mlph, respectively [9]. All three types cause skin and hair hypopigmentation; however, GS types 1 and 2 cause neurological impairment and immunological impairment, respectively, as side effects. However, with Mlph mutation, only depigmentation is reportedly apparent and no other side effects are observed [10-12]. Therefore, Mlph could be an important target gene for depigmentation studies.

The roles of many uncharacterized miRNAs in the skin have been studied. For example, miR-31 enhances cell proliferation and migration in keratinocytes, and aids in skin wound healing [13]. A previous study showed that miR-218 decreased melanogenesis by directly inhibiting microphthalmia-associated transcription factor (MITF) in melan-a mouse melanocytes [14]. Recent studies targeted the 3'-UTR of genes associated with melanosome transport. For example, miR-203 significantly suppressed the growth of human and canine melanoma cells and inhibited melanosome transport through the suppression of MITF and Rab27a expressions [15]. In addition, miR-145 reportedly decreases MyoVa and FSCN1 expressions in human melanoma cells [16]. However, miRNAs that only target Mlph among the motor proteins associated with melanosome transport in mouse melanocytes have not yet been reported. In this study, we attempted to find miRNAs that regulate Mlph expression.

\section{Results}

\subsection{Identification of miRNAs Targeting Mlph in Mice}

To identify miRNA targeting Mlph in mice, we used two programs. One was TargetScan, and the other was DIANA-microT v3.0. These two online bioinformatics programs predicted miRNAs that could directly target the $3^{\prime}$-UTR of Mlph. In all, 265 miRNAs were predicted by TargetScan and 17 by DIANA-microT v3.0. We synthesized six miRNAs and inhibitors (Inh) identified by both programs (Figure 1). The microRNA sequences are shown in Table 1.

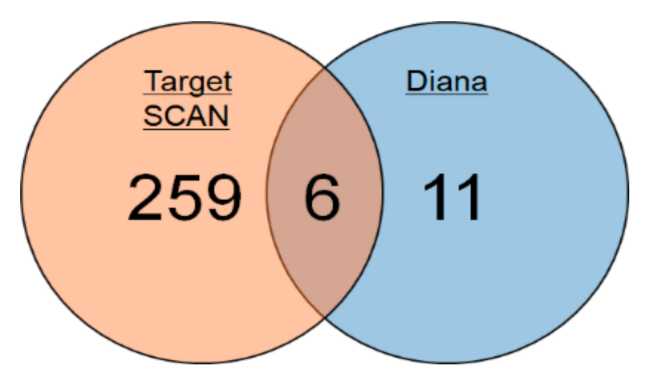

Figure 1. Prediction of miRNAs targeting melanophilin (Mlph) in mice.

Table 1. Sequences of microRNAs used in this study.

\begin{tabular}{cc}
\hline microRNA & Sequence \\
miR-1839-5p & 5'-AAGGUAGAUAGAACAGGUCUUG-3' \\
miR-5110 & miR-3082-5p \\
miR-342-5p & 5'-GGAGGAGGUAGAGGGUGGUGGAAUU-3' \\
miR-3090-3p & 5'-GACAGAGUGUGUGUGUCUGUGU-3' \\
miR-186-5p & 5'-AGGGGUGCUAUCUGUGAUUGAG-3' \\
miR-1839-5p Inh. & 5'-UCCCAGGUGACACCCUGACUCA-3' \\
miR-5110 Inh. & 5'-CAAAGAAUUCUCCUUUUGGGCU-3' \\
miR-3082-5p Inh. & 5'-CAAGACCUGUUCUAUCUACCUU-3' \\
miR-342-5p Inh. & 5'-AAUUCCACCACCCUCUACCUCCUCC-3' \\
miR-3090-3p Inh. & 5'-ACACAGACACACACACUCUGUC-3' \\
miR-186-5p Inh. & 5'-CUCAAUCACAGAUAGCACCCCU-3' \\
\hline
\end{tabular}




\subsection{Effect of miRNAs on Mlph Expression in Melan-a Melanocytes}

To investigate whether the six synthesized miRNAs affect melanosome transport, melan-a mouse melanocytes were treated with $10 \mathrm{nM}$ of the miRNAs. After $72 \mathrm{~h}$ transfection, we found that melanosome aggregation was induced. Three miRNAs, miR-342-5p, miR-3082-5p, and miR-1839-5p, induced melanosome aggregation in melan-a melanocytes. However, the other three did not induce melanosome aggregation (Figure 2a). Among the six miRNAs, miR-342-5p had the strongest inhibitory effect on the Mlph protein expression level compared to the negative control (Figure 2b).

A
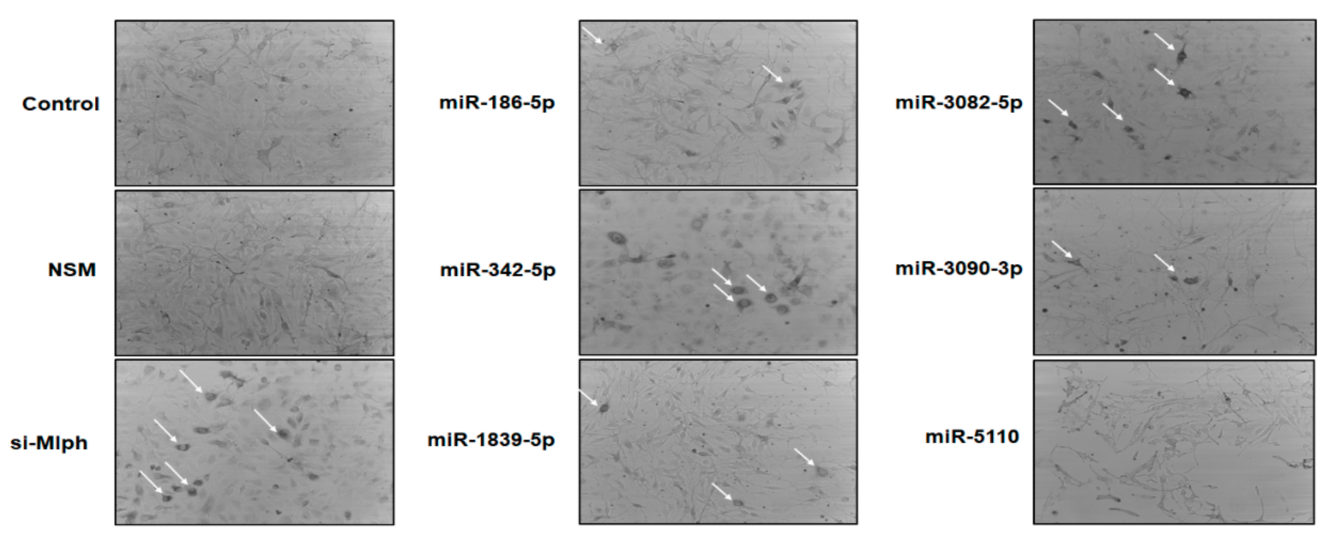

B

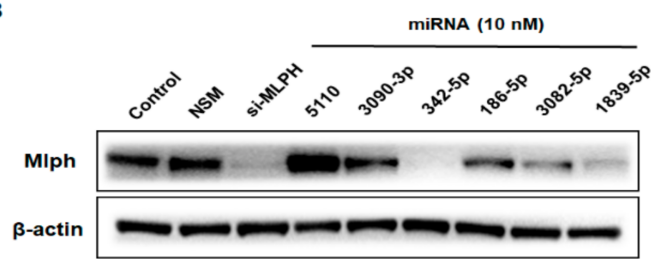

Figure 2. Effect of miRNAs in melan-a melanocytes. Melan-a melanocytes were seeded at $3 \times 10^{5}$ cells/well in $10 \pi$ dishes in RPMI-1640 media with $10 \%$ FBS(Fetal Bovine Serum), $1 \% \mathrm{P} / \mathrm{S}$, and $200 \mathrm{nM}$ TPA. (A) After $24 \mathrm{~h}$, melan-a melanocytes were treated with miRNAs. We examined cells transfected with six representative microRNAs. After $72 \mathrm{~h}$, the cells were observed in bright field using a digital microscope (iRiS ${ }^{\mathrm{TM}}$, Logosbio, Anyang, Korea). Melanosome aggregation was estimated by counting cells with perinuclear melanosome aggregation in three random microscopic fields per well at 200× magnification. (B) Melan-a melanocytes treated with $10 \mathrm{nM}$ miRNAs for $72 \mathrm{~h}$. Six miRNAs showed effects on protein expression level from Mlph. Values are presented as the mean \pm SD from three wells.

\subsection{Effect of miR-342-5p on Mlph Expression in Melan-a Melanocytes}

To investigate whether miR-342-5p affected melanosome transport in a dose-dependent manner,

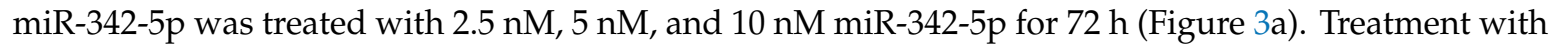
$2.5 \mathrm{nM}, 5 \mathrm{nM}$, and $10 \mathrm{nM}$ miR-342-5p increased melanosome aggregation in a dose-dependent manner compared with control. miR-342-5p decreased the mRNA and protein levels of Mlph in a dose-dependent manner (Figure 3b,c). The mRNA Mlph expression level was measured using quantitative real-time PCR. 
A

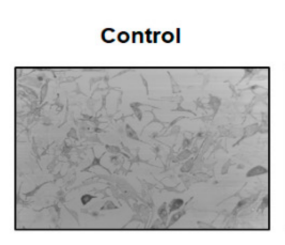

B
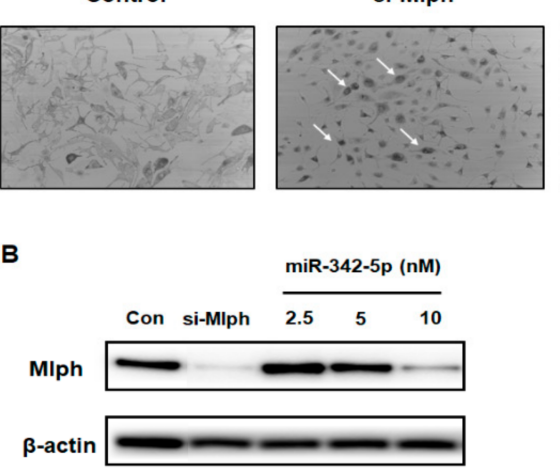

D
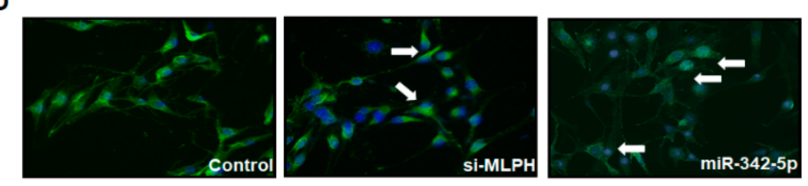

Figure 3. Effects of miR-342-5p in melan-a melanocytes. Melan-a melanocytes were seed at $3 \times 10^{5}$ cells/well in 10 $\pi$ dishes in RPMI-1640 media with 10\% FBS, 1\% P/S, 200 nM TPA. (A) After 24 h, melan-a melanocytes were treated with miR-342-5p in a dose-dependent manner. After transfection for $72 \mathrm{~h}$, the cells were observed in bright field using a digital microscope (iRiS ${ }^{\mathrm{TM}}$, Logosbio, Korea). (B) Melan-a melanocytes treated with $10 \mathrm{nM}$ miRNAs for $72 \mathrm{~h}$. miR-342-5p effects on the level of Mlph. (C) Cells were harvested for RNA $48 \mathrm{~h}$ after transfection. Mlph expression levels were determined using real-time q-PCR. The experiment was repeated three times. Data were analyzed using Student's un-paired $t$-test ${ }^{* *}, p<0.01$. (D) Melan-a melanocytes were seeded at $2 \times 10^{5}$ cells/chamber. After $24 \mathrm{~h}$, melan-a melanocytes were treated with miRNA and siRNA according to the manufacturer's protocol. After $72 \mathrm{~h}$, cells were photographed. Values are presented as the mean \pm SD from three wells.

The aggregation of melanosomes around the nucleus was imaged using HMB45 antibody immunofluorescence (Figure 3d).

\subsection{Effect of mir-342-5p on the Rab27a and MyoVa Expressions}

To determine whether miR-342-5p affects other key melanosome transport protein expressions, we examined Rab27a and MyoVa expressions together. miR-342-5p decreased the level of Mlph but did not affect the levels of MyoVa and Rab27a. To check the specificity of the effect of miR-342-5p on Mlph expression, the rescue effect was examined using an inhibitor of miR-342-5p (Figure 4a). The inhibitor of miR-342-5p increased the levels of Mlph. 
A



C

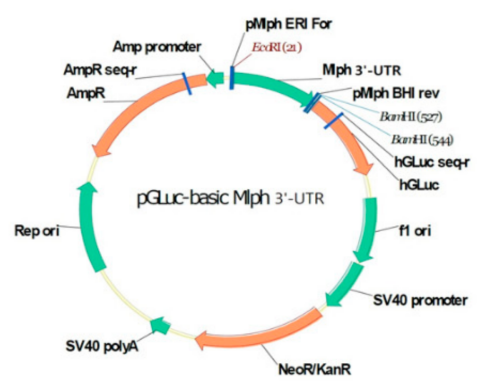

B


Figure 4. Mlph, MyoVa, and Rab27a expression levels by miR-342-5p or with inhibitors. Melan-a melanocytes were seeded at $3 \times 10^{5}$ cells in six-well plates for $24 \mathrm{~h}$. (A) After $24 \mathrm{~h}$, melan-a melanocytes were treated with miR-342-5p and inhibitors. After $72 \mathrm{~h}$, miR-342-5p effected the levels of Mlph, MyoVa, and Rab27a. (B) We examined whether mmu-miR-342-5p directly targets Mlph by using online miRNA target prediction databases. (C) Luciferase reporters were linked with Mlph 3'-UTRs. miR-342-5p or scrambled mimic (Scr mimic) were co-transfected with a luciferase-UTR construct into melan-a melanocytes, and luciferase activity was determined.

\section{5. miR-342-5p Directly Targets Mlph in Melan-a Melanocytes}

We identified the binding site of mmu-miR-342-5p in the 3'-UTR of Mlph (Figure 4b). To identify the specificity of miR-342-5p, we constructed a sensor vector by joining the $3^{\prime}$-UTR region of mouse Mlph to a luciferase reporter pGLuc-basic vector. To determine the target sequence recognized by the miRNA, miR-342-5p was treated with an inhibitor, while the control was not. Treatment with miR-342-5p reduced luciferase activity, and treatment of miR-342-5p with inhibitor restored the luciferase activity (Figure 4c).

\section{Discussion}

Melanosomes are fully matured during transport from the melanocyte nucleus to the melanocyte dendrite tip. Then, melanosomes are transferred to the keratinocytes [17,18]. During melanosome transport, three proteins, Mlph, Rab27a, and MyoVa, form a tripartite complex. These are the key regulators of melanosome transport $[19,20]$.

In this study we identified miRNAs that affected Mlph expression, and attempted to understand their effect. When the melan-a melanocytes were treated with the selected miRNAs, the miRNAs were observed to suppress melanosome transport (Figure 2a). Significant aggregation of melanosomes in the perinuclear area was evident in the transfection of miR-342-5p in melan-a melanocytes (Figure 3a). miR-342-5p decreased the protein levels of Mlph in a dose-dependent manner (Figure 3b). Previous studies have showed that miR-5110 suppresses pigmentation in alpaca melanocytes by co-targeting MLPH and WNT family member 1 [21]. However, miR-5110 has no effect on melanosome transport in melan-a melanocytes. miR-342-5p decreased the levels of Mlph, but did not affect Rab27a and MyoVa expressions (Figure 4a). Until now there has been no report on the miRNA targeting Mlph in mice or humans. From this study, we found miRNA that affects the expression of Mlph in 
mice, and we can expect miRNAs with similar function in humans, which is an area of research to be explored in future studies. According to several previous studies, Mlph expression is very important for melanosome transport. Mutations in Mlph have been shown to cause defects in melanosome transport in melanocytes [7]. Treatment with miR-342-5p reduced the mRNA level of Mlph (Figure 3c) by targeting the 3'-UTR of Mlph.

miR-3082-5p and miR-1839-5p downregulated Mlph and Rab27a expression levels (data not shown). There are reports that one miRNA can target two genes simultaneously. For example, a study published in Japan in 2013 showed that one miRNA can target two genes simultaneously in a gastric cancer cell line [22]. miR-224 targets DPYSL2 (dihydropyri-midinase-like-2) and KLAS, which is a proto-oncogene [22]. DPYSL2 encodes the collapsin response mediator protein 2 (CRMP2), a cytosolic protein [23]. This protein is necessary for the growth and promotion of neurons. It is usually tethered to the cell membrane, and is essential for tissue signaling. Therefore, miR-3082-5p and miR-1839-5p can be considered to target two genes simultaneously, similar to miR-224. miR-3082-5p is reportedly controlled in mouse melanoma cells by curcumin and could be a potential anti-cancer miRNA [24]. However, miR-1839-5 $p$ has not yet been investigated.

It has been reported that miR-342-5p is upregulated in Alzheimer's disease (AD) transgenic mouse models, including APP/PS1, PS1DE9, and PS1-M146V lines [25]. miR-342-5p binds directly to the 3'-UTR of AnkG mRNA and decreases AnkG levels through translation repression in AD transgenic mouse neurons. However, there have been no reports on the effects of miR 342-5p on Mlph expression. We found a novel miRNA that affects Mlph and melanosome transport and could be important for understanding Mlph expression and skin pigmentation regulation.

\section{Materials and Methods}

\subsection{Materials}

miRNA was purchased from GenePharma (Shanghai, China). Lipofectamine RNAi MAX was purchased from Invitrogen (Carlsbad, CA, USA).

\subsection{Cell Culture}

Melan-a melanocytes are highly pigmented, immortalized murine melanocytes derived from C57BL/6 mice. Melan-a melanocytes were obtained from Dr. Dorothy Bennett (St. George's Hospital, London, UK). Melan-a melanocytes were maintained in RPMI-1640 medium (Welgene, Gyeongsan, Korea) supplemented with $10 \%$ fetal bovine serum (FBS), $10 \mathrm{U} / \mathrm{mL}$ penicillin, $100 \mu \mathrm{g} / \mathrm{mL}$ streptomycin, and 200 nM phorbol 12-myristate 13-acetate (PMA; Sigma-Aldrich Co., St. Louis, MO, USA).

\subsection{Transfection}

One day before transfection, melan-a melanocytes were seeded in six-well plates at $60 \%$ confluency, and miRNAs, inhibitors, and $20 \mathrm{nM}$ siRNA mixed RNAi MAX (Invitrogen) were applied according to the manufacturer's protocol.

\subsection{Western Blotting}

Blots were incubated with $\beta$-actin antibody (1:20000, Sigma), MyoVa antibody (1:800, Cell Signaling Technology, Beverly, MA, USA), Rab27a antibody (1:500, Santa Cruz, CA, USA), Mlph antibody (1:800, ProteinTech Group, Inc. Chicago, IL, USA) at $4{ }^{\circ} \mathrm{C}$ overnight. Blots were then washed three times with TBS-T (Tris Buffered Saline with Tween 20) and incubated with horseradish peroxidase-conjugated anti-rabbit (1:1000, Bethyl Laboratories, Montgomery, AK, USA) or anti-mouse (1:20000, Bio-Rad, Hercules, CA, USA) antiserum at room temperature for one hour. Bound antibodies were detected using a SuperSignal $® W e s t$ Pico Chemiluminescent Substrate (Thermo Scientific, Waltham, MA, USA). The bands on the membranes were detected via chemiluminescence and visualized with FluorChem $\mathrm{E}$ (ProteinSimple, San José, CA, USA). 


\subsection{Immuno-Fluorescence (IF)}

One day before transfection, melan-a melanocytes were seeded in one chamber at $60 \%$ confluency and miRNA plus $20 \mathrm{nM}$ siRNA mixed RNAi MAX (Invitrogen) were applied according to the manufacturer's protocol. Melan-a melanocytes transfected with miRNA and siRNA after $72 \mathrm{~h}$ were washed with DPBS (Dulbecco's Phosphate-Buffered Salin) and then were fixed in $2 \mathrm{~mL}$ formalin for 15 minutes at room temperature. Chambers were washed three times with PBS and were then permeabilized with $1 \mathrm{~mL}$ of $0.1 \%$ Tripton for 15 minutes and then blocked using $1 \mathrm{~mL}$ of $2 \%$ BSA(Bovine Serum Albumin). After thorough washing, sections were incubated with first antibody (HMB45) diluted with $2 \%$ BSA for two to three days at $4{ }^{\circ} \mathrm{C}$ with shaking. FITC-conjugated antibody was diluted $2 \%$ BSA or PBS treated for one hour at $4{ }^{\circ} \mathrm{C}$ with shaking followed by washing three times with PBS. The cells were stained with 4'-6-diamidino-2-phenylindole DAPI) for 15 minutes at room temperature.

\subsection{Quantitative Real-Time PCR}

Quantitative real-time PCR was performed using a FastStart Essential DNA Probe Master kit (Roche, Mannheim, Germany) with the Universal Probe Library (Roche). The reaction was carried out according to the manufacturer's protocol. The probes for Rab27a (\#21, NM_023635.5), Mlph (\#108, NM_053015.3), and MyoVa (\#21, NM_010864.2) were designed using the Probe Library Assay Design Center. The cycling conditions were 600 seconds at $95^{\circ} \mathrm{C}$ followed by 40 cycles at $95^{\circ} \mathrm{C}$ for 20 seconds and $60{ }^{\circ} \mathrm{C}$ for 40 seconds on a Lightcycler®Nano (Roche, Basel, Switzerland). The resulting cDNA was amplified with the following primers: Rab27a sense 5'-GAAGACCAGAGGGCAGTGAA-3', and antisense 5'-ACTGGTTTCAAAA-TAGGGGATTC-3'; MyoVa sense 5'-GCGCCATCACCCTAAACA-3', and antisense 5' $5^{\prime}$ CCAGTTGACTGACATTGTACCTG-3'. The three gene expression levels were normalized to mouse $\beta$-actin.

Values are presented as mean \pm SD from three wells.

\section{7. miRNA and Small Interfering RNAs}

To investigate potential miRNAs targeting melanophilin, we used two programs, TargetScanMouse (http://www.targetscan.org) and Diana Tools (http://diana.imis.athenainnovation.gr/DianaTools/index.php).

All miRNA used in this study, including inhibitors for each microRNA, were purchased from GenePharma (Shanghai, China) (Table 1). siRNA oligonucleotides were purchased from Bioneer (Daejeon, Korea). Sense and antisense sequences for individual duplexes targeting mouse $M l p h$ were as follows: Mlph sense was 5'-GGGCAAAAUACAAAAGGAGUUTT-3', and antisense was 5'CUCCUUUUGUAUUUUGCCCUUTT-3'.

\subsection{Detection and Quantification of Melanosome Aggregation}

Melan-a melanocytes were seeded in 24-well plates and cultured for $24 \mathrm{~h}$. The cells were then rinsed in DPBS and treated with samples of RPMI- 1640 containing 2\% FBS for three days. The cells were observed under bright field using a digital microscope (iRiS ${ }^{\mathrm{TM}}$, Logosbio, Korea). Evaluation of melanosome aggregation was performed by counting cells with perinuclear melanosome aggregates in three random microscopic fields per well at 200× magnification.

Values are presented as mean \pm SD from three wells.

\subsection{Luciferase Assay}

We constructed a sensor vector by joining the regions with a possible binding site from the $3^{\prime}$-UTR of mouse Mlph to a luciferase reporter pGLuc-basic vector purchased from Cosmogenetech (Seoul, Korea) in order to identify the target sequences recognized by the miRNAs. For amplification of these mRNAs, melan-a melanocytes were seeded in six-well plates at a concentration of $3.0 \times 10^{5} /$ well the day before the transfection. The vector, at a concentration of $0.5 \mu \mathrm{g} / \mathrm{well}$, and $10 \mathrm{nM}$ miRNAs or 
nonspecific control miRNA was used for the co-transfection of the cells using the cationic liposomes Lipofectamine RNAi MAX. Luciferase activities were measured using a BioLux Luciferase Assay Kit (NEW ENGLAND BioLabs Inc., MA, USA) according to the manufacturer's protocol, $48 \mathrm{~h}$ after the cotransfection.

Author Contributions: Conceptualization: S.J. and J.S.; validation: C.H., J.I., and S.C.; formal analysis: J.A and J.I.; investigation: S.J and J.A; data curation: J.A.; writing—original draft preparation: S.J. and J.S.; writing-review and editing: J.A and J.S.; visualization: J.I.; supervision: J.S.; project administration: J.S.

Funding: This research received no external funding.

Acknowledgments: This work was supported by a National Research Foundation of Korea (NRF) grant funded by the Korea government (MSIT)(NRF-2017R1D1A1B03030045).

Conflicts of Interest: The authors declare that there are no conflicts of interest.

\section{References}

1. Gillbro, J.M.; Olsson, M.J. The melanogenesis and mechanisms of skin-lightening agents—Existing and new approaches. Int. J. Cosmet. Sci. 2011, 33, 210-221. [CrossRef] [PubMed]

2. Thody, A.J.; Higgins, E.M.; Wakamatsu, K.; Ito, S.; Burchill, S.A.; Marks, J.M. Pheomelanin as well as eumelanin is present in human epidermis. J. Invest. Dermatol. 1991, 97, 340-344. [CrossRef] [PubMed]

3. Cichorek, M.; Wachulska, M.; Stasiewicz, A.; Tyminska, A. Skin melanocytes: Biology and development. Postepy Dermatol. I. Alergol. 2013, 30, 30-41. [CrossRef] [PubMed]

4. Schiaffino, M.V. Signaling pathways in melanosome biogenesis and pathology. Int. J. Biochem. Cell Biol. 2010, 42, 1094-1104. [CrossRef] [PubMed]

5. Park, J.I.; Lee, H.Y.; Lee, J.E.; Myung, C.H.; Hwang, J.S. Inhibitory effect of 2-methyl-naphtho[1,2,3-de] quinolin-8-one on melanosome transport and skin pigmentation. Sci. Rep. 2016, 6, 29189. [CrossRef] [PubMed]

6. Chia, W.J.; Tang, B.L. Emerging roles for Rab family GTPases in human cancer. Biochim. Biophys. Acta 2009, 1795, 110-116. [CrossRef] [PubMed]

7. Matesic, L.E.; Yip, R.; Reuss, A.E.; Swing, D.A.; O'Sullivan, T.N.; Fletcher, C.F.; Copeland, N.G.; Jenkins, N.A. Mutations in Mlph, encoding a member of the Rab effector family, cause the melanosome transport defects observed in leaden mice. Proc. Natl. Acad. Sci. USA 2001, 98, 10238-10243. [CrossRef]

8. Griscelli, C.; Durandy, A.; Guy-Grand, D.; Daguillard, F.; Herzog, C.; Prunieras, M. A syndrome associating partial albinism and immunodeficiency. Am. J. Med. 1978, 65, 691-702. [CrossRef]

9. Malhotra, A.K.; Bhaskar, G.; Nanda, M.; Kabra, M.; Singh, M.K.; Ramam, M. Griscelli syndrome. J. Am. Acad. Dermatol. 2006, 55, 337-340. [CrossRef]

10. Aslan, D.; Sari, S.; Derinoz, O.; Dalgic, B. Griscelli syndrome: description of a case with Rab27A mutation. Pediatr. Hematol. Oncol. 2006, 23, 255-261. [CrossRef]

11. Menasche, G.; Ho, C.H.; Sanal, O.; Feldmann, J.; Tezcan, I.; Ersoy, F.; Houdusse, A.; Fischer, A.; de Saint Basile, G. Griscelli syndrome restricted to hypopigmentation results from a melanophilin defect (GS3) or a MYO5A F-exon deletion (GS1). J. Clin. Invest. 2003, 112, 450-456. [CrossRef] [PubMed]

12. Westbroek, W.; Klar, A.; Cullinane, A.R.; Ziegler, S.G.; Hurvitz, H.; Ganem, A.; Wilson, K.; Dorward, H.; Huizing, M.; Tamimi, H.; et al. Cellular and clinical report of new Griscelli syndrome type III cases. Pigment Cell Melanoma Res. 2012, 25, 47-56. [CrossRef] [PubMed]

13. Li, D.; Li, X.I.; Wang, A.; Meisgen, F.; Pivarcsi, A.; Sonkoly, E.; Stahle, M.; Landen, N.X. MicroRNA-31 Promotes Skin Wound Healing by Enhancing Keratinocyte Proliferation and Migration. J. Invest. Derm. 2015, 135, 1676-1685. [CrossRef] [PubMed]

14. Guo, J.; Zhang, J.F.; Wang, W.M.; Cheung, F.W.; Lu, Y.F.; Ng, C.F.; Kung, H.F.; Liu, W.K. MicroRNA-218 inhibits melanogenesis by directly suppressing microphthalmia-associated transcription factor expression. Rna Biol. 2014, 11, 732-741. [CrossRef] [PubMed]

15. Noguchi, S.; Kumazaki, M.; Mori, T.; Baba, K.; Okuda, M.; Mizuno, T.; Akao, Y. Analysis of microRNA-203 function in CREB/MITF/RAB27a pathway: Comparison between canine and human melanoma cells. Vet. Comp. Oncol. 2016, 14, 384-394. [CrossRef] [PubMed] 
16. Dynoodt, P.; Speeckaert, R.; De Wever, O.; Chevolet, I.; Brochez, L.; Lambert, J.; Van Gele, M. miR-145 overexpression suppresses the migration and invasion of metastatic melanoma cells. Int. J. Oncol. 2013, 42, 1443-1451. [CrossRef]

17. Wasmeier, C.; Hume, A.N.; Bolasco, G.; Seabra, M.C. Melanosomes at a glance. J. Cell Sci. 2008, 121, $3995-3999$. [CrossRef]

18. Mackintosh, J.A. The antimicrobial properties of melanocytes, melanosomes and melanin and the evolution of black skin. J. Theor. Biol. 2001, 211, 101-113. [CrossRef]

19. Van Gele, M.; Geusens, B.; Schmitt, A.M.; Aguilar, L.; Lambert, J. Knockdown of myosin Va isoforms by RNAi as a tool to block melanosome transport in primary human melanocytes. J. Invest. Derm. 2008, 128, 2474-2484. [CrossRef]

20. Van Den Bossche, K.; Naeyaert, J.M.; Lambert, J. The quest for the mechanism of melanin transfer. Traffic 2006, 7, 769-778. [CrossRef]

21. Yang, S.; Liu, B.; Ji, K.; Fan, R.; Dong, C. MicroRNA-5110 regulates pigmentation by cotargeting melanophilin and WNT family member 1. FASEB J. 2018, 32, 5405-5412. [CrossRef] [PubMed]

22. Hashimoto, Y.; Akiyama, Y.; Yuasa, Y. Multiple-to-multiple relationships between microRNAs and target genes in gastric cancer. PLoS ONE 2013, 8, e62589. [CrossRef] [PubMed]

23. Pham, X.; Song, G.; Lao, S.; Goff, L.; Zhu, H.; Valle, D.; Avramopoulos, D. The DPYSL2 gene connects mTOR and schizophrenia. Transl. Psychliatry 2016, 6, e933. [CrossRef] [PubMed]

24. Dahmke, I.N.; Backes, C.; Rudzitis-Auth, J.; Laschke, M.W.; Eidinger, P.; Menger, M.D.; Meese, E.; Mahlknecht, U. Curcumin intake affects miRNA signature in murine melanoma with mmu-miR-205-5p most significantly altered. PLoS ONE 2013, 8, e81122. [CrossRef] [PubMed]

25. Sun, X.; Wu, Y.; Gu, M.; Zhang, Y. miR-342-5p decreases ankyrin G levels in Alzheimer's disease transgenic mouse models. Cell Rep. 2014, 6, 264-270. [CrossRef] [PubMed]

(C) 2019 by the authors. Licensee MDPI, Basel, Switzerland. This article is an open access article distributed under the terms and conditions of the Creative Commons Attribution (CC BY) license (http://creativecommons.org/licenses/by/4.0/). 